\title{
Anticoagulation in pregnant women with mechanical heart valve prostheses
}

\author{
S S Meschengieser, C G Fondevila, M T Santarelli, M A Lazzari
}

\begin{abstract}
Objective-To evaluate the outcome of pregnancy in women with mechanical heart valve prostheses in relation to the anticoagulant treatment used in the first trimester and the incidence of thrombotic and bleeding complications.

Methods-92 pregnancies in 59 women were followed between 1986 and 1997. In 31 pregnancies, oral anticoagulants were discontinued when pregnancy was diagnosed and subcutaneous heparin was started (12 $500 \mathrm{U}$ every 12 hours) adjusted to prolong the adjusted partial thromboplastin time to twice the control level. In the second trimester oral anticoagulants were resumed but changed to heparin again 15 days before the expected delivery date. In 61 pregnancies oral anticoagulants were continued during the first trimester. The same regimen of heparin was used for delivery.
\end{abstract}

Results-Abortion or fetal losses were similar $(p=0.5717)$ in women exposed to oral anticoagulants in the first trimester $(13 / 61 ; 25 \%)$ compared with those who received adjusted subcutaneous heparin $(6 / 31 ; 19 \%)$. Embolic episodes were more common $(p=0.0029)$ in women who received heparin $(4.92 \%)$ compared with those on oral anticoagulants $(0.33 \%)$. Embolic episodes were cerebral and transient. No valve thromboses were observed. No malformations appeared in the 71 newborns, except for one case of hydrocephalus. There were no maternal deaths secondary to thrombotic complications. The only death was the result of major bleeding after the delivery of a premature stillborn.

Conclusions-Oral anticoagulants seem to be safer for the mother than adjusted subcutaneous heparin. Heparin does not offer a clear advantage over oral anticoagulation in the pregnancy outcome. (Heart 1999;82:23-26)

Keywords: pregnancy; oral anticoagulants; heparin; prosthetic valves

The use of oral anticoagulants during pregnancy is controversial and there is no agreement on the most suitable treatment for patients with mechanical prosthetic heart valves, who have a high risk of thromboembolism.

Coumarin derivatives carry the risk of embryopathy when given between the sixth and the 12 th week of pregnancy, ${ }^{1}$ but this complica- tion can be prevented when heparin is substituted for oral anticoagulants. The reports of coumarin embryopathy vary widely, from $0 \%{ }^{2-6}$ up to $29.6 \%$ in a study by Iturbe Alessio et $a l^{7}$ in which malformations were mostly aesthetic and overdiagnosis by geneticists has been proposed. The estimated risk of malformation is now believed to be less than $5 \%{ }^{8}$

Both oral anticoagulants and heparin carry the risk of fetal loss, probably from different mechanisms. Heparin can induce retroplacental haemorrhage and although its administration during the first trimester can prevent the occurrence of malformations, it certainly does not improve the outcome of the pregnancy.

Pregnancy induces several changes in the haemostatic system resulting in a hypercoagulability state with higher risk of thromboembolism. Recently the efficacy of heparin in preventing thrombotic complications in patients with prosthetic heart valves has been questioned in a retrospective study by Sbarouni and Oakley ${ }^{9}$ and in a report by Salazar et $a l .{ }^{10}$

We evaluated the outcome of 92 pregnancies in 59 patients with prosthetic heart valves with either adjusted doses of subcutaneous heparin or oral anticoagulation in the first trimester, and we assessed the incidence of thrombotic complications with each regimen.

\section{Methods}

STUDY PATIENTS

We followed 92 consecutive unselected pregnancies in 59 patients with prosthetic heart valves between the beginning of 1986 and June 1997. The age of the patients ranged from 17 to 46 years, mean (SD) 27.7 (6.4) (table 1). Twenty one women had more than one pregnancy (12 women had two pregnancies, six had three pregnancies, and three had four pregnancies). There was a mean (SD) interval between surgery and pregnancy of 4.86 (3.63) years (range 0 to 17). One patient underwent aortic valve replacement while pregnant and another one had her prostheses changed in the second trimester of pregnancy.

SITE AND TYPE OF PROSTHESES

There were 39 women with mitral valve prostheses $(66 \%, 95 \%$ confidence interval (CI) 52.5 to 77.6$), 15$ with aortic valve prostheses (25\%, 95\% CI 15.4 to 39$)$, two with tricuspid valve prostheses $(3 \%, 95 \%$ CI 0.59 to 13$)$, and three with double valve replacement (mitralaortic) (5\%, 95\% CI 1.32 to 15.06$)$ (table 1).

Most of the valves were of ball cage type (34/ $62,55 \% ; 95 \%$ CI 41.7 to 67.3$) ; 23$ (37\%) were 
Table 1 Features at study entry

\begin{tabular}{lll}
\hline Age (years) (mean (SD)) & $27.7(6.4)$ & \\
$\quad$ Range & 17 to 46 & \\
Valve position (n = 59) & 39 & $66 \%$ \\
Mitral & 15 & $25 \%$ \\
Aortic & 2 & $3 \%$ \\
Tricuspid & 3 & $5 \%$ \\
Double (mitral-aortic) & & \\
Valve type (n = 62) & 34 & $55 \%$ \\
Ball caged valves & 23 & \\
Mitral & 11 & \\
Aortic & 23 & \\
Tilting disc & 16 & \\
Mitral & 5 & \\
Aortic & 2 & \\
Tricuspid & 5 & \\
Bileaflet valves & 3 & \\
Mitral & 2 & \\
Aortic & & \\
\hline
\end{tabular}

tilting disc valves (95\% CI 25.4 to 50.3 ), and five $(8 \%)$ were bileaflet valves ( $95 \%$ CI 3.0 to 18.5).

\section{ANTICOAGULATION}

All women were receiving acenocoumarol as their oral anticoagulant. In 31 pregnancies, oral anticoagulation was discontinued when the pregnancy was confirmed and subcutaneous heparin was started with an initial dose of $12500 \mathrm{U}$ every 12 hours. The dose was adjusted to prolong the adjusted partial thromboplastin time (APTT) to twice the control level by four hours after administration. Laboratory controls including platelet counts were performed every seven to 10 days. Heparin was given for a mean (SD) of 39.58 (18.74) days (range 15 to 110 ). Three patients received only two weeks of heparin and one received 20 days. The rest of them received heparin for at least four weeks. The subcutaneous heparin was continued until the end of the first trimester, when oral anticoagulation was resumed. The international normalised ratio (INR) was determined every three weeks. Two to three weeks before the estimated date of delivery, heparin was resumed and if a caesarean delivery was planned, the last heparin dose was given 12 hours before it and resumed 12 hours afterwards. In case of a vaginal delivery, heparin was stopped when labour started, but this situation was more difficult to standardise.

In 61 pregnancies oral anticoagulation was not discontinued in the first trimester because the pregnancy was confirmed too late. For delivery the same regimen of subcutaneous heparin was employed.

In 38 of the pregnancies, the women were maintained with an INR of 2.5 to 3.5 while being treated with oral anticoagulants by concurrent administration of low dose aspirin. ${ }^{11}$ Otherwise the therapeutic range was 3 to 4 . The daily dose of acenocoumarol at the start of the pregnancy was calculated.

\section{THROMBOEMBOLISM}

Thromboembolic episodes were defined as a temporary or permanent neurological deficit of sudden onset without any other explanation. They were considered as major (permanent deficit) or minor (reversible neurological deficit).
BLEEDING

Bleeding was classified as major when it was fatal or required transfusions.

FETAL OUTCOME

Newborn infants were examined by trained neonatologists aware of the changes induced by oral anticoagulants, to determine whether malformations were present.

STATISTICAL ANALYSIS

Data are presented as means and 95\% confidence intervals. Where appropriate, Fisher's exact test or the $\chi^{2}$ test was used. A $p$ value less than 0.05 was considered significant.

\section{Results}

In the group of women who continued with oral anticoagulation in the first trimester, pregnancy resulted in 46 liveborn infants $(75 \%$, $95 \%$ CI 62.4 to $85.1 ; 34$ vaginal deliveries and 12 caesarean sections). In the group who received adjusted subcutaneous heparin in the first trimester, there were 25 liveborn infants (81\%, 95\% CI 61.9 to 91.2 ; 11 vaginal deliveries and 14 caesarean sections) (table 2).

Fetal loss predominated in the first trimester in the women receiving heparin $(5 / 6 ; 95 \% \mathrm{CI}$ 6.1 to 34.5$)$. In women receiving oral anticoagulants the frequency of fetal loss was similar in the first and second trimester $(6 / 15 ; 95 \% \mathrm{CI}$ 4.06 to 20.8 ) and lower in the third trimester (3/15; 95\% CI 1.3 to 14.6$)$ (table 3). Although the rate of fetal loss was higher in the group of women who continued on oral anticoagulation, the difference was not significant $(\mathrm{p}=0.5717)$ (table 3).

FETAL OUTCOME

The weight of the newborn infants ranged from $1850 \mathrm{~g}$ to $4000 \mathrm{~g}$ (median weight $3000 \mathrm{~g}$ ). Thirteen of the 71 neonates were of low birth weight (below $2500 \mathrm{~g}$ ). There were no neonatal deaths. One baby developed a hydrocephalus. The mother of that baby received oral anticoagulants during the first trimester and she went into premature labour while she was still orally anticoagulated. She had a vaginal delivery of a low birth weight baby, although we would have preferred a caesarean section. The mother received fresh frozen plasma to prevent bleeding. We are not certain about the origin of the hydrocephalus but we cannot rule out intracranial bleeding as a cause.

No malformations were diagnosed in the remaining 45 newborn infants who were exposed to oral anticoagulants during the first trimester (table 3). The mean (SD) daily dose of acenocoumarol at the beginning of pregnancy was $3.23(1.30) \mathrm{mg}$, range 0.71 to 7.0 . In 10 cases the daily dose was $5 \mathrm{mg}$ or more. Abortions or fetal loss in the women exposed to higher doses of acenocoumarol were not significantly more common than in the group receiving less than $5 \mathrm{mg}(30 \%$ v $23 \%$, $\mathrm{p}=0.968)$.

\section{EMBOLIC COMPLICATIONS}

Four cerebral embolic episodes were observed (4.92/100 patient-months) while the patients 
Table 2 Outcome of pregnancies, by treatment in the first trimester

\begin{tabular}{|c|c|c|c|c|c|c|}
\hline & \multicolumn{3}{|c|}{ Oral anticoagulant } & \multicolumn{3}{|c|}{ Adjusted heparin } \\
\hline & $n$ & $\%$ & $(95 \% C I)$ & $n$ & $\%$ & $(95 \% C I)$ \\
\hline Pregnancies & 61 & & & 31 & & \\
\hline \multicolumn{7}{|l|}{ Liveborn infants } \\
\hline Healthy babies & 45 & 74 & (60.7 to 83.8 ) & 25 & 81 & (61.9 to 91.2$)$ \\
\hline Hydrocephalus & 1 & & & 0 & & \\
\hline Low birth weight & 7 & 11 & (5.1 to 23 ) & 6 & 19 & (8.12 to 38$)$ \\
\hline \multicolumn{7}{|l|}{ Deliveries } \\
\hline Vaginal & 34 & 56 & (42.5 to 68 ) & 11 & 35 & (20 to 55$)$ \\
\hline Caesarean & 12 & 20 & (11 to 32$)$ & 14 & 45 & (27.8 to 64$)$ \\
\hline
\end{tabular}

CI, confidence interval.

Table 3 Abortions or fetal wastage, by anticoagulant treatment during the first trimester

\begin{tabular}{|c|c|c|c|c|c|c|c|}
\hline \multirow[b]{2}{*}{ Trimester } & \multicolumn{3}{|c|}{ Oral anticoagulant $(n=61)$} & \multicolumn{4}{|c|}{ Adjusted heparin $(n=31)$} \\
\hline & $n$ & $\%$ & $(95 \% \mathrm{CI})$ & $n$ & $\%$ & $(95 \% C I)$ & $p$ Value \\
\hline First & 6 & 10 & (4.06 to 20.8 ) & 5 & 16 & (6.1 to 34.5 ) & \\
\hline Second & 6 & 10 & (4.06 to 20.8 ) & - & & & \\
\hline Third & 3 & 5 & (1.3 to 14.6$)$ & 1 & 3 & (0.17 to 18.5$)$ & \\
\hline Total & 15 & 25 & (14.8 to 37.6$)$ & 6 & 19 & (8.12 to 38$)$ & 0.5717 \\
\hline
\end{tabular}

CI, confidence interval.

Table 4 Embolic complications

\begin{tabular}{llll}
\hline & Oral anticoagulant & Adjusted heparin & p Value \\
\hline Follow up (months) & 588.3 & 81.2 & 0.0029 \\
Embolic episodes (n) & 2 & 4 & \\
$\quad$ Minor & 0 & 0 & \\
$\quad$ Major & & & \\
Incidence (episodes/100 & patient-months) & 0.33 (CI 0.06 to 1.36) & 4.92 (CI 1.58 to 12.8) \\
$\quad$ Site of valve & 1 & 1 & \\
$\quad$ Mitral & 1 & 1 & \\
$\quad$ Aortic & 0 & 0 & \\
Valve thrombosis & & &
\end{tabular}

were receiving subcutaneous heparin. In three of the four episodes, the APTT was prolonged into the target therapeutic range. In one case the APTT was inadequately prolonged. All episodes were cerebral and transient. Two transient cerebral emboli were associated with oral anticoagulant treatment $(0.33 / 100$ patient-months). In one of these cases the INR was 3.91 and in the other it was 1.8. Thus only one episode can be explained by inadequate anticoagulation. There was a significant difference $(p=0.0029)$ between embolism associated with oral anticoagulants (two episodes in 588.3 months) and with heparin treatment (four episodes in 81.2 months).

The six embolic episodes occurred in four women (three mitral valve prostheses and one aortic); two women had two embolic complications each, one during oral anticoagulation and the other during subcutaneous heparin. The aortic prosthesis was a Bjork type; the mitral prostheses were two ball caged and one $\mathrm{St}$ Jude. One of the women had a third embolic episode in the same pregnancy, which was explained by infective endocarditis. She was switched to subcutaneous heparin for seven days at 24 weeks of pregnancy until tomographs ruled out haemorrhage and the antibiotic treatment was considered adequate. No valve thrombosis was observed (table 4 ).

BLEEDING COMPLICATIONS

We had one episode of fatal bleeding in a patient with systemic lupus erythematosus who delivered a dead fetus at 30 weeks of pregnancy while she was on oral anticoagulants. We had no information on the INR from the time of admission and the bleeding could not be stopped. There were two minor episodes of bleeding: a haematoma in a caesarean section wound and an episode of postpartum bleeding associated with heparin administration. No transfusion was necessary in either case and the outcomes were satisfactory.

\section{Discussion}

The management of anticoagulation during pregnancy is controversial and there is no ideal treatment. Patients with prosthetic heart valves have the highest thromboembolic risk, and pregnancy itself is a thrombogenic situation owing to physiological hypercoagulation. Modern valves have fewer thromboembolic complications because of better design and materials, but there are many women with earlier models of prosthetic valve attending our anticoagulation clinic who become pregnant.

There is general agreement that, where possible, oral anticoagulants should be discontinued in the first trimester to avoid the warfarin embryopathy. However, there is no agreement on the dose or on the way the heparin should be administered. Low fixed doses of subcutaneous heparin have proven to be insufficient to prevent embolism. Our experience until 1986 was similar to that reported by Lee et al and Chen et al. ${ }^{12}{ }^{13}$ Our incidence of embolic complications with low dose heparin ${ }^{14}$ was higher (11.7 episodes/100 patient-months) than with oral anticoagulation or adjusted heparin. Adjusted subcutaneous heparin decreased the number of embolic episodes to $4.92 \%$, although the lowest incidence $(0.33 \%)$ is associated with oral anticoagulation. In our experience, however, the administration of adjusted subcutaneous heparin was not as disappointing as reported by Salazar et al and Sbarouni and Oakley. ${ }^{9}{ }^{10}$ We had no maternal deaths from valve thrombosis or cerebral embolism, and the embolic episodes left no sequelae. The single death in our series was explained by major bleeding after the delivery of a dead fetus. Embolism was more common with mitral valve prostheses and with adjusted heparin. However, the fact that two women each had two embolic episodes with either treatment suggests an individual thrombogenic predisposition or a thrombogenic valve. In Sbarouni's report, ${ }^{9}$ heparin was blamed for the high rate of valve thrombosis and maternal deaths. However, that study was retrospective, different centres were involved, and the adequacy of heparin anticoagulation was not reported in every case where a thrombotic complication was observed. It is difficult to assume that subcutaneous adjusted heparin is unsafe when the level of anticoagulation cannot be established. In Salazar's report, ${ }^{10}$ adjusted heparin again resulted in poor protection from valve thrombosis and embolism, and in that study laboratory control was adequate and heparin was given more frequently to overcome the high rate of thrombotic complications, but without success. We think Salazar's patients are 
comparable with our population, so the reasons for their poor results are not clear. Ginsberg et al concluded that the high frequency of adverse effects with heparin may be associated with maternal comorbid conditions. ${ }^{15}$ Ginsberg and Hirsh also suggested that patients with mechanical heart valves may be resistant to moderate doses of heparin and that an inadequate target therapeutic range can explain the reported failure. ${ }^{16}$

The protection given by subcutaneous unfractionated heparin is certainly not constant and probably there are a few hours each day during which anticoagulation is inadequate. Intravenous heparin is an alternative but requires hospital admission. This may be appropriate before delivery but is not ideal in the first trimester. The use of low molecular weight heparin has been reported in pregnancy, ${ }^{17}$ and this may represent the future management of these patients.

The incidence of abortions or fetal wastage is rather high in this population of pregnant patients with prosthetic heart valves. There have been reports of abortion rates as high as $50 \%{ }^{12}$ but most range between $23 \%$ and $33 \%$. $^{3}{ }^{4}{ }^{1013}$ Switching to heparin in the first trimester certainly does not dramatically improve the number of successful pregnancies. In our own experience the rate of fetal loss before 1986 was as high as $40 \%$, but it has since fallen to $25 \%$, probably because of better obstetric and cardiological care. The use of oral anticoagulants or heparin might be directly involved in first trimester fetal losses, but it seems more difficult to explain fetal wastage during the second or third trimester, when obstetric conditions are more likely to be responsible.

Cotrufo et al found no abortions or malformations in a group of patients whose warfarin requirement was less than $5 \mathrm{mg} /$ day. ${ }^{18}$ There are no reports on acenocoumarol dose and the incidence of abortion or embryopathy. We did not observe any significant difference in fetal outcome between those women requiring $5 \mathrm{mg}$ or more of acenocoumarol a day and those receiving less than $5 \mathrm{mg}$.

No malformations were observed either in the neonates exposed to oral anticoagulants in the first trimester or in those receiving heparin. Central nervous system abnormalities resulting from either haemorrhage or malformation have been found to be related to exposure to coumarin derivatives during the second and third trimester of pregnancy, ${ }^{19}{ }^{20}$ although they are uncommon. We had one case of hydrocephalus in a low birth weight baby born to a woman exposed to oral anticoagulants during her entire pregnancy; her baby was delivered while she was still on coumarins. We cannot be certain about the cause of the hydrocephalus but intrauterine or perinatal intracranial haemorrhage cannot be ruled out. We agree with Salazar et al that a caesarean section is indicated if labour starts while the mother is still on oral anticoagulants, as reversal of the mother's anticoagulation can be quickly achieved. ${ }^{10}$

There is no ideal method of anticoagulation during pregnancy in women with prosthetic valves, and no definitive recommendations can be made at present. We prefer to avoid oral anticoagulants during the first trimester and before delivery, although they appear to be safer for the mother. The outcome of pregnancy may be slightly improved when heparin is given in the first trimester but this did not reach statistical significance in our study. Low molecular weight heparins may represent the future management of these patients, as they do not cross the placenta, induce less osteoporosis, and offer more consistent protection against thromboembolism.

We are indebted to Olga Suárez and Verónica Rodríguez for their assistance in writing this paper.

1 Hall JG, Pauli RM, Wilson KM. Maternal and fetal sequelae of anticoagulation during pregnancy. $\mathrm{Am} f \mathrm{Med}$ 1983;68:122-40.

2 Ben Ismail M, Abid F, Trabelsi S, et al. Cardiac valve prostheses, anticoagulation and pregnancy. Br Heart f 1986;55: $101-5$.

3 Sarelli P, England MJ, Berk MR, et al. Maternal and fetal sequelae of anticoagulation during pregnancy in patients with mechanical heart valve prostheses. Am f Cardiol 1989; 63:1462-5.

4 Born D, Martinez EE, Almeida PAM, et al. Pregnancy in patients with prosthetic heart valves. The effect of anticoagulation on mother, fetus and neonate. Am Heart $\mathcal{F}$ 1992;124:413-17.

5 Pavankumar P, Venugopal P, Kaul U, et al. Pregnancy in patients with prosthetic cardiac valves. A 10 years

6 Larrea JL, Nuñez L, Reque JA, et al. Pregnancy and mechanical valve prostheses: a high risk situation for the mechanical valve prostheses: a high risk situation for the

7 Iturbe Alessio J, Fonseca MC, Mutchinik O, et al. Risks of anticoagulant therapy in pregnant women with artificial anticoagulant therapy in pregnant women with
heart valves. $N$ Engl f Med 1986;315:1390-3.

8 Oakley CM. Anticoagulants in pregnancy. Br Heart $\mathcal{F} 1995$; 74:107-11.

9 Sbarouni E, Oakley CM. Outcome of pregnancy in women Sbarouni E, Oakley CM. Outcome of pregnancy in
with valve prostheses. Br Heart $\mathcal{F} 1994 ; 71: 196-201$.

10 Salazar E, Izaguirre R, Verdejo JG, et al. Failure of adjusted doses of subcutaneous heparin to prevent thromboembolic phenomena in pregnant patients with mechanical cardiac valve prostheses. F Am Coll Cardiol 1996;27:1698-703.

11 Meschengieser S, Fondevila CG, Frontroth JP, et al. Low intense oral anticoagulation plus low dose aspirin versus high intense oral anticoagulation alone: a randomized trial in patients with mechanical prosthetic heart valves. $\mathcal{f}$ Thorac Cardiovasc Surg 1997;113:910-16.

12 Lee PK, Wang RYC, Cho JSF, et al. Combined use of warfarin and adjusted heparin during pregnancy in patients with artificial valves. F Am Coll Cardiol 1986;8:221-4.

13 Chen WWC, Chan CS, Lee PK, et al. Pregnancy in patients with prosthetic heart valves: an experience with 45 pregnancies. $Q \mathcal{F} \mathrm{Med}$ 1982;203:358-65.

14 Meschengieser S, Fondevila C, Lazzari MA. Anticoagulation in pregnancy in patients with prosthetic heart valves [abstract]. Rev Invest Clin (suppl) 1994:215.

15 Ginsberg JS, Hirsh J, Turner DC, et al. Risks to the fetus of anticoagulant therapy during pregnancy. Thromb Haemost 1989;61:197-203.

16 Ginsberg JS, Hirsh J. Use of antithrombotic agents during pregnancy. Chest 1995;108(suppl):305-11S.

17 Lee LH, Liauw PCY, Ng ASH. Low molecular weight heparin for thromboprophylaxis during pregnancy in 2
patients with mechanical mitral valve replacement. Thromb Haemost 1996;76:628-30.

18 Cotrufo M, de Luca TS, Calabro R, et al. Coumarin anticoagulation during pregnancy in patients with mechanical

valve prostheses. Eur 7 Cardiothorac Surg 1991;5:300-5.
19 Palacios-Macedo X, Díaz-Devis C, Escudero J. Fetal risk with the use of coumarin anticoagulant agents in pregnant patient with intracardiac ball valve prosthesis. Am $\mathcal{F}$ Cardiol 1969;24:853-6.

20 Stevenson RE, Burton M, Ferlauto GJ, et al. Hazards of oral anticoagulants during pregnancy. $f A M A$ 1980;243:154951 